\title{
BMJ Open A systematic review of economic evaluations of seasonal influenza vaccination for the elderly population in the European Union
}

\author{
Gemma E Shields, Jamie Elvidge, Linda M Davies
}

To cite: Shields GE, Elvidge J, Davies LM. A systematic review of economic evaluations of seasonal influenza vaccination for the elderly population in the European Union. BMJ Open 2017;7:e014847. doi:10.1136/ bmjopen-2016-014847

- Prepublication history and additional material is available. To view please visit the journal (http://dx.doi.org/ 10.1136/ bmjopen-2016-014847).

Received 20 0ctober 2016 Revised 12 December 2016 Accepted 18 April 2017

CrossMark

Centre for Health Economics, University of Manchester, Manchester, UK

Correspondence to Gemma E Shields; gemma. shields@manchester.ac.uk

\section{ABSTRACT}

Objectives The Council of the European Union (EU) has recommended that action should be taken to increase influenza vaccination in the elderly population. The aims were to systematically review and critically appraise economic evaluations for influenza vaccination in the elderly population in the EU.

Methods Electronic searches of the NHS Economic Evaluation, Health Technology Assessment, MEDLINE and Embase databases were run to identify full economic evaluations. Two levels of screening were used, with explicit inclusion criteria applied by two independent reviewers at each stage. Prespecified data extraction and critical appraisal were performed on identified studies. Results were summarised qualitatively.

Results Of the 326 search results, screening identified eight relevant studies. Results varied widely, with the incremental cost-effectiveness ratio ranging from being both more effective and cheaper than no intervention to costing $€ 459350$ per life-year gained. Cost-effectiveness was most sensitive to variations in influenza strain, vaccination type and strategy, population and modelling characteristics.

Conclusions Most studies suggest that vaccination is cost-effective (seven of eight studies identified at least one cost-effective scenario). All but one study used economic models to synthesise data from different sources. The results are uncertain due to the methods used and the relevance and robustness of the data used. Sensitivity analysis to explore these aspects was limited. Integrated, controlled prospective clinical and economic evaluations and surveillance data are needed to improve the evidence base. This would allow more advanced modelling techniques to characterise the epidemiology of influenza more accurately and improve the robustness of costeffectiveness estimates.

\section{INTRODUCTION}

Seasonal influenza is a highly contagious acute viral infection, with a risk of complications and mortality. It is important to policy makers because it has a large economic impact, both in terms of costs and population quality of life. ${ }^{1}$ The prevalence of influenza varies each season and is affected by multiple factors (eg, virus strength and climate). It is a
Strengths and limitations of this study

- This study systematically reviewed economic evaluations for influenza vaccination in the elderly population in the EU, a practice that is recommended in policy.

- To the authors' knowledge, this search brings the only previous systematic review of economic evidence for influenza vaccination up to date.

- The study summarises and critically appraises the current evidence base and also discusses potential avenues for future research.

- The review was limited to English-language studies; there is scope to broaden the search to include other languages and identify further papers.

serious public health concern generally and particularly for high-risk groups such as older people. ${ }^{2-7}$ In the European Union (EU), the elderly population makes up $18.9 \%$ of the population, which is over 96 million people (2015 figures). ${ }^{8}$

In the elderly population with influenza-like illness (ILI), hospitalisation risk has been reported to be up to $8.8 \%$, with up to $4.2 \%$ admitted cases requiring time in an intensive care unit and a risk of death when admitted to hospital of $3.1 \%-13.5 \% .^{5}$ While complication rates are lower in the elderly population compared with other high-risk groups, older people are more likely to have chronic conditions and fall into multiple highrisk groups (eg, heart failure, Parkinson's disease and asthma). ${ }^{67}$ In addition, influenza exacerbates existing chronic conditions (eg, respiratory conditions) and causes secondary infections (such as pneumonia) that are more frequently recorded as the cause of death. ${ }^{9}$ These factors mean that influenza may contribute to more severe illnesses and be a causal factor of increased mortality risk, rather than the primary recorded cause of death (laboratory testing for the presence of 
influenza infection is uncommon). ${ }^{9}{ }^{10}$ Statistical modelling suggests that influenza may be responsible for between $2.5 \%$ and $8.1 \%$ of deaths in the over-75 age group. ${ }^{9}$

Vaccines are the most commonly used intervention to prevent influenza; they work by simulating infection, provoking the body to create antibodies to protect against infection. ${ }^{11}$ Two types are available: trivalent which protects against three viruses and quadrivalent which protects against four viruses. Vaccines can also contain squalene-based adjuvants (eg, MF59) which aim to improve the efficacy of vaccines. The Council of the EU recommended that by $2014-2015,75 \%$ of the elderly population should be vaccinated against influenza. ${ }^{12}$ The latest published (2012) figures show that the EU mean vaccination coverage is around $44.7 \%$ (range $1 \%$ to $77.4 \%$ ) of the elderly population, which is substantially short of the EU target. ${ }^{13}$

In the elderly population, the aims of vaccination are to reduce the rate of influenza and to decrease the burden of complications (morbidity and mortality) resulting from influenza, although evidence of protection is limited and uncertain in this population. ${ }^{11}{ }^{14} \mathrm{In}$ the adult population aged 18-65, influenza (relative reduction in influenza risk following vaccination) has been reported to have an efficacy rate of $59 \%$ (95\% CI 51 to 67$)$ in a pooled analysis of 12 seasons. ${ }^{14}$ As people age, the immune system can become compromised and less responsive to vaccination. ${ }^{15}$ When focusing specifically on the elderly population, effectiveness appears to be much lower, with one study reporting $23 \%$ efficacy for elderly individuals living in care homes in seasons where vaccine matching (the degree of similarity between the circulating virus and strain in the vaccine) is good. ${ }^{16}$ For individuals living in the community, the same study noted that vaccines were not statistically significantly effective at preventing influenza, although well-matched vaccines provided benefits in reducing related admissions to hospital and pneumonia. ${ }^{16}$ In addition, when comparing effectiveness evidence across populations, evidence in the elderly population is much more reliant on low-quality studies (eg, non-randomised cohort studies with small samples). ${ }^{1416}$

It is important that intervention strategies, such as vaccination programmes, provide value for money in budget-constrained healthcare systems. As such, in light of the recommendations for influenza vaccination in the elderly population and in an environment with an ageing population and the issues discussed above around vaccination effectiveness, relevant economic evaluations are likely to be of interest to policy makers.

This study had two aims: first, to determine whether influenza vaccination interventions were demonstrated to be cost-effective in older people in the EU. The second aim was to critically appraise the methods and data to evaluate the validity and robustness of the study findings, to inform policy makers and future research.

\section{METHODS}

A systematic literature search and narrative review was conducted to identify economic evaluations of influenza vaccinations in the elderly population in the EU.

\section{Search strategy}

Search terms across the databases used included disease-specific terms, for example, 'influenza'; terms for the intervention, for example, 'vaccination'; and terms for economic evaluations, for example, 'cost-effectiveness'. Search terms and strategies varied slightly according to the database design and functionality. Free-text and standardised subject terms were used. Alternative spellings were included to capture all potentially relevant citations. The search strategy was piloted to ensure it identified all known studies and all studies identified in a previous review. An example search strategy is provided in the online supplementary material.

An initial search was run in November 2014, using the NHS Economic Evaluation Database (EED) and the Health Technology Assessment database (via The Cochrane Library). At the time of initial search, the EED was a reliable source for economic evaluations, using a very precise search over a wide number of databases to capture relevant studies which helps to reduce the number of irrelevant study results. ${ }^{17}$ NHS EED ceased being updated in March 2015, so the updated search (November 2015) included the MEDLINE, EMBASE and Econlit databases. The NHS EED search terms were used to identify economic evaluations. ${ }^{18}$

\section{Selection}

Identified citations were manually screened to identify potentially relevant papers to include in the review, using explicit pre-defined inclusion criteria: the study sample or modelled population had to be (1) based in the EU and (2) aged $\geq 60$ or $\geq 65$ years (the definition of elderly used in influenza policies across Europe). Studies did not need to exclusively look at this age group but had to present the results for the elderly population separately to other populations if they did consider wider populations. Studies had to compare the seasonal influenza vaccination intervention to a comparator intended to reduce the burden of illness associated with influenza (eg, an alternative form of vaccination or antiviral treatments) or usual care/no intervention. This meant that the comparator arm could also include a proportion of people who were vaccinated against influenza. A fourth inclusion criteria was that studies had to be full economic evaluations, producing an incremental cost-effectiveness ratio using a health outcome. No restriction was placed on publication date. The review was limited to English-language articles. After screening of titles and abstracts, full papers were retrieved and screened using the same inclusion criteria.

Two reviewers independently screened citations and full papers, with a third reviewer consulted to resolve any disagreements. The primary reason for study exclusion was recorded at each stage. 


\section{Data extraction}

Comprehensive data extraction was undertaken, in line with the NHS EED handbook and with some key additions on modelling technique from a review of economic evaluations of vaccinations. ${ }^{19} 20$ Predefined data extraction and quality assessment forms (see online supplementary material) were used to extract information on study methodology, results, limitations, evidence gaps and quality for critical appraisal. Two reviewers extracted data independently; results were compared and discussion, with any disagreements settled by a third reviewer.

Cost figures were converted into 2014 euros for presentation using the price index for each country and the purchasing power parity conversion factor to facilitate comparison between studies in different EU countries. ${ }^{21} 22$

\section{Data synthesis}

Extracted data were summarised in text and tables. Study characteristics, cost and outcome data were tabulated and summarised. Critical appraisal was qualitatively synthesised with the help of the checklist. The key aspects assessed included the reporting of key information (eg, study design and how parameters were identified), methods used and the validity of cost and health benefits used.

\section{RESULTS}

\section{Summary of studies}

Figure 1 Summary of the flow of studies identified and selected for review. An overview of study setting, population and intervention/comparator is given in table 1; table 2 presents an overview of the study characteristics. A tabular overview of the critical appraisal is provided in the online supplementary material.

\section{Study results}

Key study outcomes are given in table 3.

\section{Vaccination type comparisons}

Three studies including two vaccine types. ${ }^{23-25}$ Adjuvanted vaccination, while associated with a higher cost of vaccination, was cost-effective when compared with standard vaccination as higher efficacy rates increased health benefits. ${ }^{23} 25$ Although the two studies focusing on adjuvanted vaccination identified it as being cost-effective, the incremental cost-effectiveness ratio (ICER) results were very different (dominant vs a cost per QALY gain). It is difficult to assess where the difference in the result arose from, due to a lack of reporting incremental health benefits and net costs. However, there were some key differences in the studies that could have affected this, including modelling

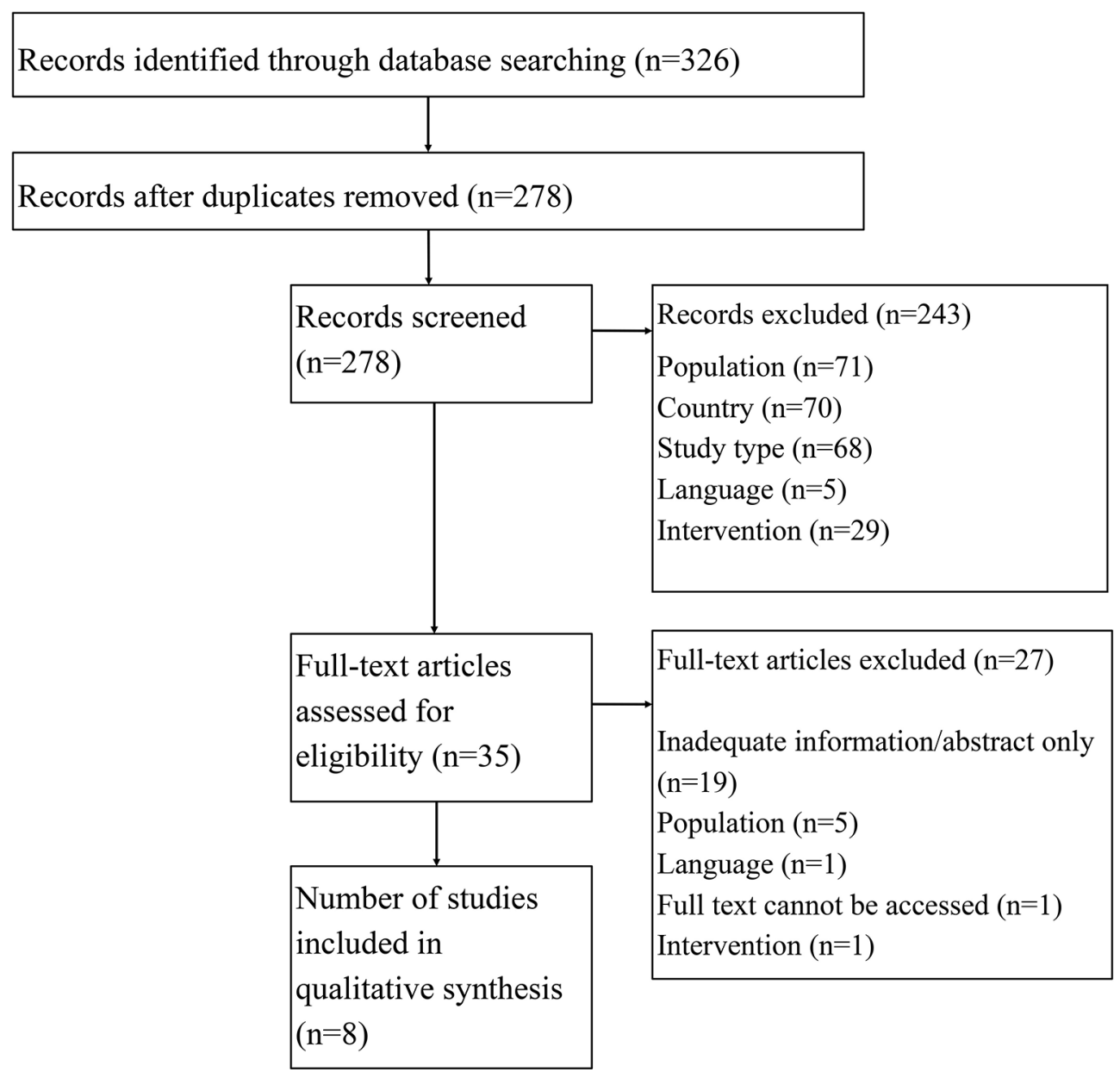

Figure 1 Flow of studies identified and selected for review. 


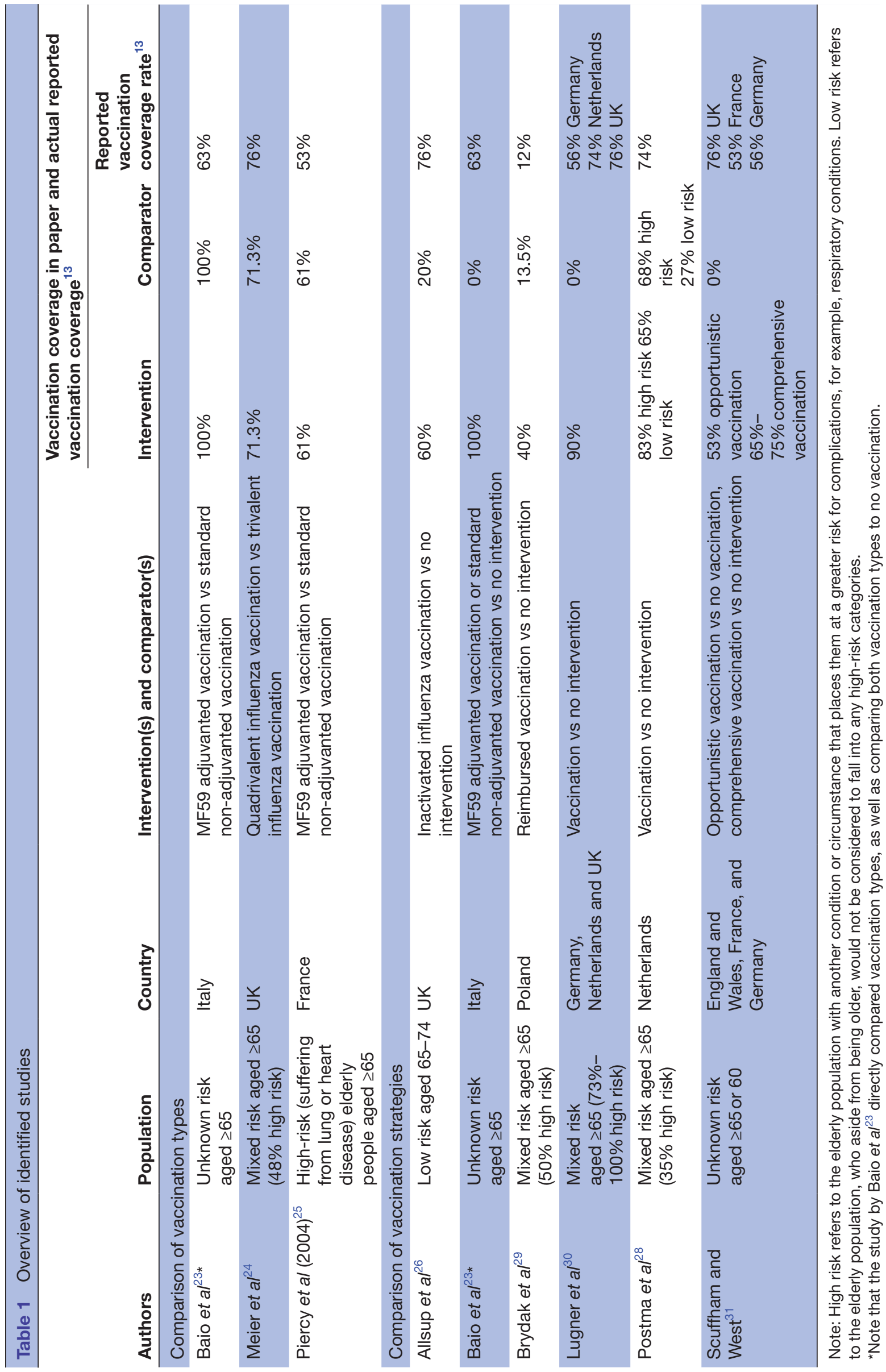

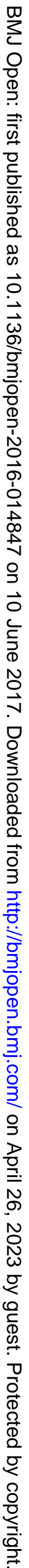




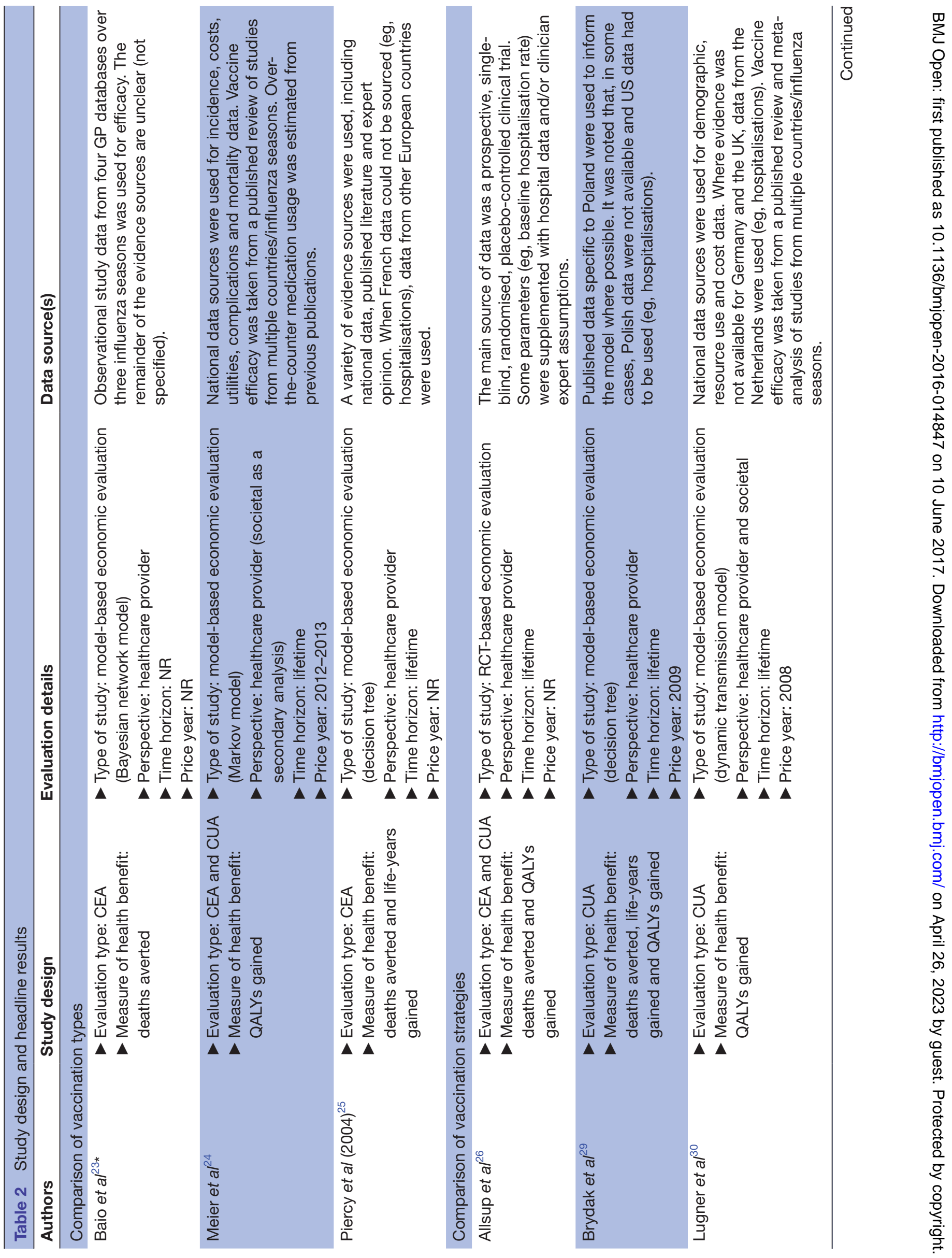




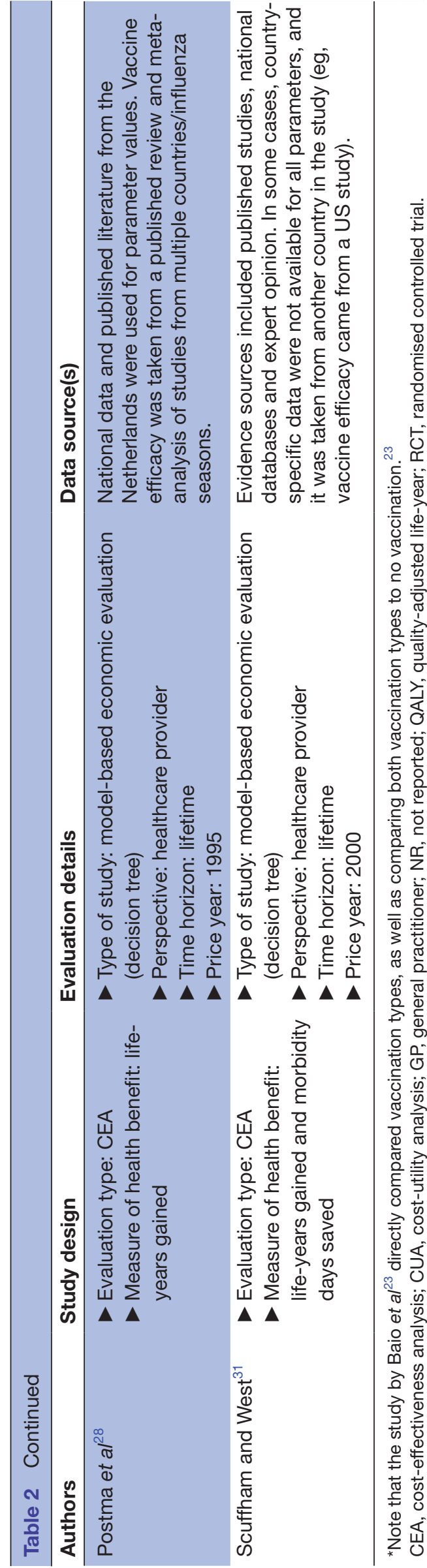

characteristics (decision tree vs Bayesian network model), the population risk level, sources of effectiveness evidence and in particular the influenza attack rate. The latter in particular stands out as being very different between studies; Piercy et al (2004) assumed a rate of 5\% (author's assumptions), whereas Baio $e t a l^{23}$ used estimates from the literature, with a mean of $16 \%{ }^{2325}$

Quadrivalent vaccination was cost-effective when compared with trivalent vaccination in the base case scenario modelled by Meier et al. ${ }^{24}$ However, this study does not make it clear whether the data used to inform efficacy of the vaccination for the base case results were specific to the elderly. The study also considered two scenarios (a best-matching season and a worst-matching season) but did not report the results separately for the elderly population.

Two studies which compared vaccination types included probabilistic sensitivity analysis. Baio $e t a t^{23}$ estimated that adjuvanted vaccination was over $90 \%$ likely to be cost-effective compared with standard vaccination. ${ }^{23}$ Meier $e t a l^{24}$ included probabilistic sensitivity analysis. ${ }^{24}$ The authors found that quadrivalent vaccination was estimated to be cost-effective in between $68 \%$ and $87 \%$ of scenarios compared with trivalent across the total modelled population. However, this was not restricted to the elderly population subgroup; thus, it is impossible to draw conclusions from this study about the uncertainty around estimates that are specific to the elderly population.

\section{Vaccination strategy comparisons}

Vaccination intervention (ie, strategies that increase the level of vaccination within a population) appears to be cost-effective throughout the studies for both the primary and sensitivity analyses conducted, with the exception of the study of Allsup et $a l^{26}$ which reported a significantly higher ICER. As previously noted, studies included different levels of vaccination in their intervention/ comparator arms; however, this does not appear to cause any systematic differences in results.

The study of Allsup $e t a l^{26}$ is noted to have significantly different results to the other studies. This study focused on the low-risk population only. In addition, it applied lower reductions in the risk of hospitalisation from vaccination and lower costs of hospitalisations. It also applied a cost of vaccination promotion where the other studies did not, increasing the validity of costs as this is likely to be needed to reach high vaccination levels. This was also the only study completed alongside a randomised controlled trial (RCT); the remainder were economic models using secondary data sources. Studies conducted within RCT have high internal validity, and this study appeared to be robust and was overall well reported. However, Jit $e t$ $a l^{27}$ noted that there are some issues when relying on one single trial for an economic evaluation has problems, in particular, a lack of external validity as the vaccine efficacy changes each season; it is possible that the study was conducted in a season in which vaccine matching was poor. $^{27}$ 


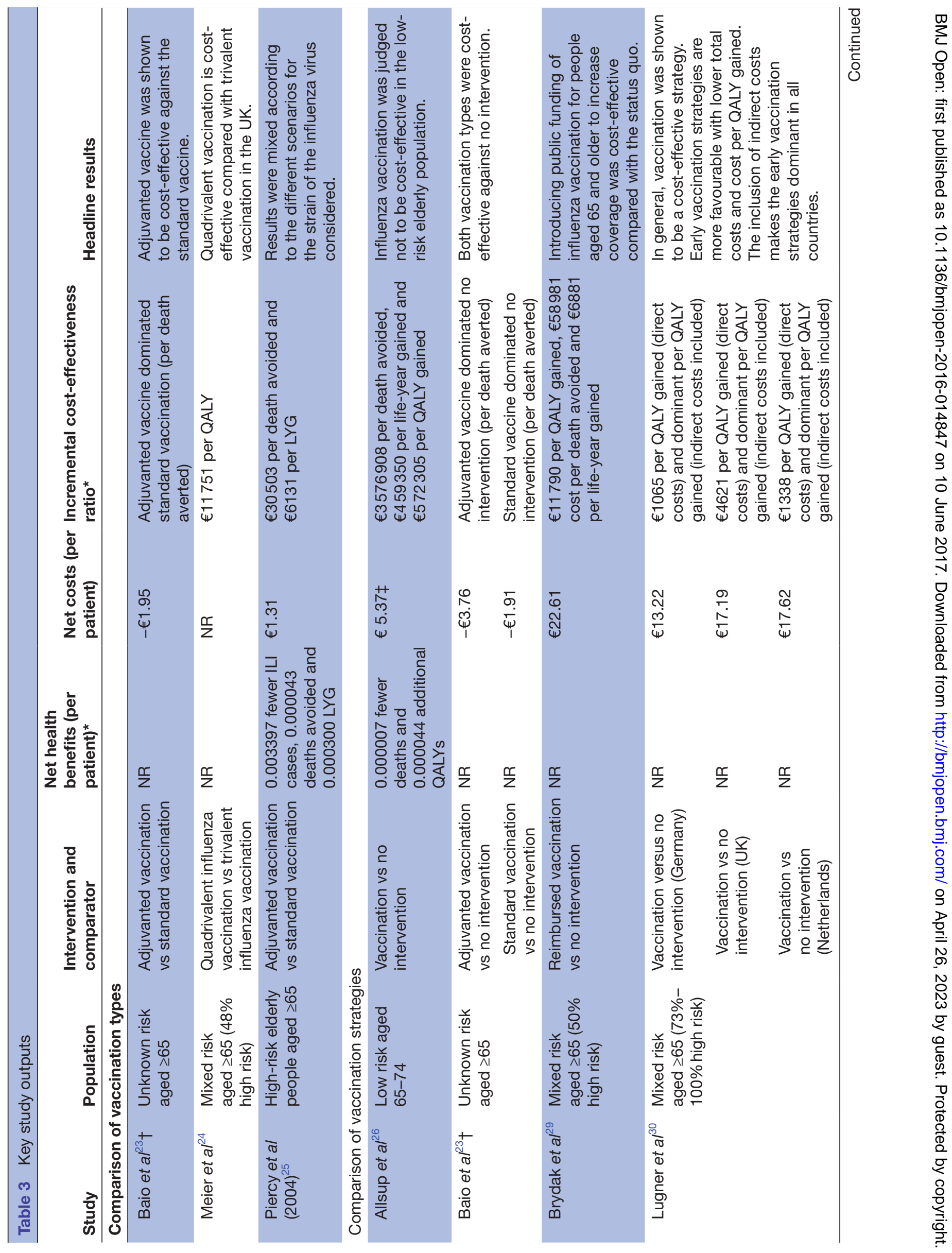




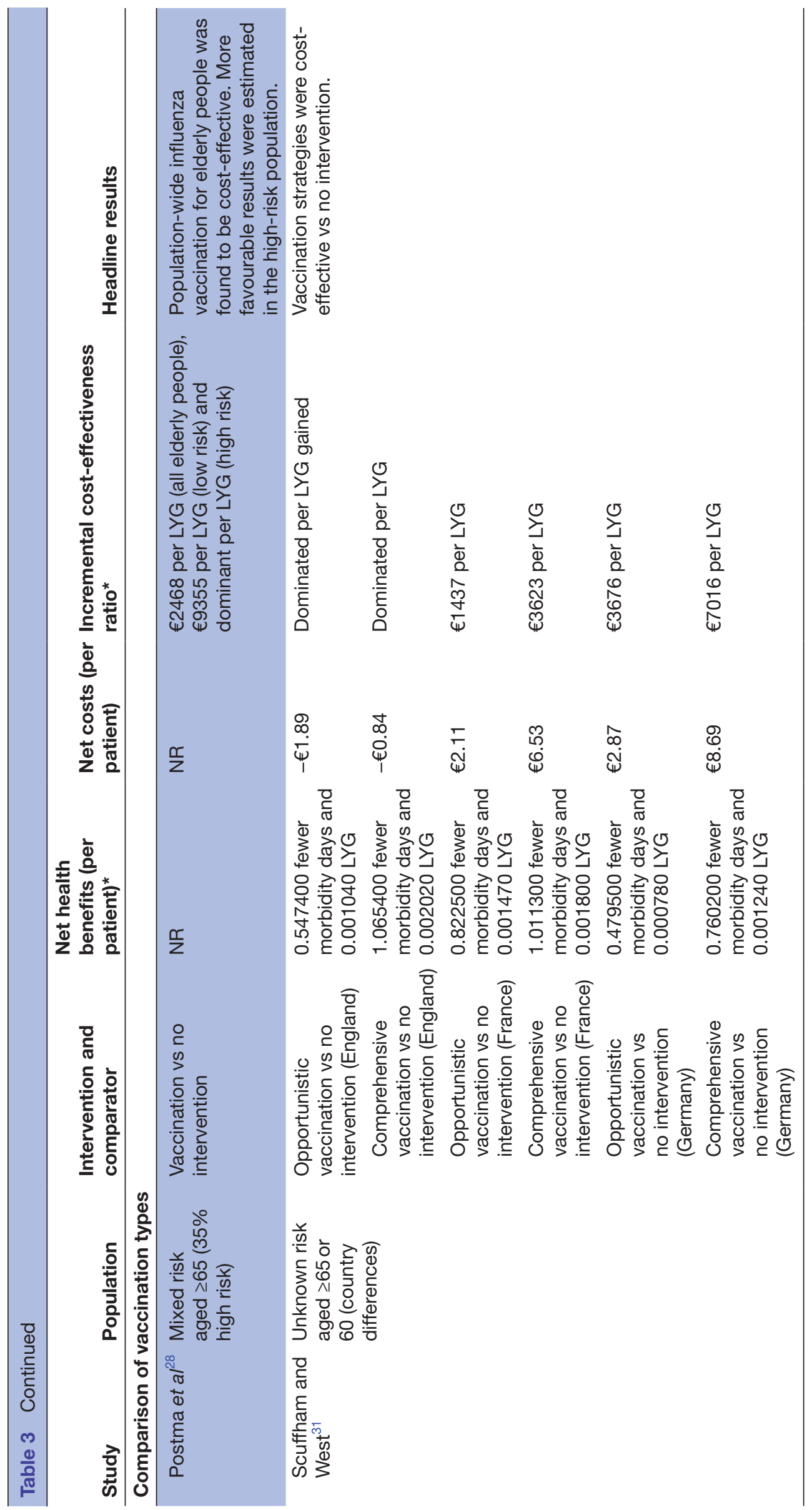

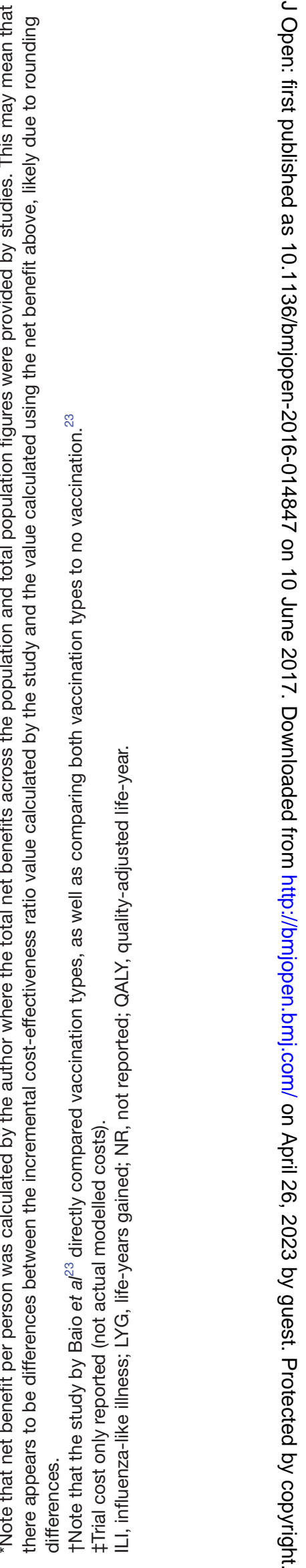


The susceptibility of the population has important implications for the rate of complications; if more people are at risk for complications, vaccination is likely to produce larger health gains. One study compared results between the low- and high-risk population. ${ }^{28}$ As expected, the vaccination of high-risk individuals was demonstrated to be more cost-effective than vaccinating low-risk individuals, as this population is more susceptible to complications, which are costly and negatively impact quality of life. ${ }^{28}$ This trend seems to be reflected in the other studies identified, for example, with Brydak $e t a l^{29}$ and Lugner $e t a l^{30}$; as the latter have a greater proportion of high-risk individuals, they have a more favourable ICER per QALY gained (in the same countries). ${ }^{29} 30$

A passive vaccination strategy was found to be more cost-effective compared with no intervention than a comprehensive/targeted strategy. ${ }^{31}$ Comprehensive strategies are associated with greater health benefits, but the passive strategy has reduced costs as they avoid the additional consultation costs, only vaccinating when people present at the general practitioner (GP) for other reasons.

One model included transmission rates and captured externalities arising from herd immunity. ${ }^{30}$ When these indirect effects were included, results became more favourable (from an ICER that was judged to be below cost-effective thresholds to dominant), as it demonstrated cost savings. The inclusion of herd immunity has important implications for the vaccination coverage in the intervention and comparator arm. Herd immunity means that the impact of increasing vaccination levels is not linear, for example, an equal change in the coverage rate between studies could have very different results depending on what the comparator/usual care coverage rate is, as the scope for benefits from herd immunity will be different. While this does not affect this review because only one study included herd immunity, it is an important point for future researchers looking to compare study results as more studies including herd immunity become available in the future.

It would be expected that different countries have comparatively different cost-effectiveness results, due to differences in healthcare service design and population differences that may affect the attack rate and complication rates. However, there does not seem to be a clear pattern demonstrated across the studies included. For example, three studies included the UK, but results are very different; one found vaccination to be cost saving and more effective (dominant), another found it to be cost increasing but cost-effective and another found it to be cost-increasing and not cost-effective. ${ }^{26}{ }^{30}{ }^{31}$ Some of this difference can be explained by assumptions made about the vaccination cost and other study differences (eg, susceptibility of the population). However, one key issue when interpreting results across countries is a lack of evidence specific to each population (discussed further in the critical appraisal section).

One study which compared vaccination to no intervention included probabilistic sensitivity analysis and determined that vaccination was $79.93 \%$ likely to be cost-effective (below the threshold of 3 GDP per capita) ${ }^{29}$ Across all studies (irrespective of intervention and comparator), scenario and one-way sensitivity analysis demonstrated key variables to be the incidence of influenza, vaccine efficacy, discount rates and complication rates (mortality and hospitalisation). In general, studies showed that despite parameter uncertainty, the majority of tested scenarios still demonstrated vaccination to be cost-effective. Further detail on sensitivity analysis conducted is provided in an overview table in the online supplementary material.

\section{Critical appraisal}

Studies were critically appraised to determine the overall quality of the evidence base; a summary is given in the online supplementary material. None of the studies reported all information required for data extraction or to conform to the WHO guidelines or recommended checklists for economic evaluations. ${ }^{19}{ }^{32-34}$ In some cases, there was a failure to report fundamental assumptions, design and inputs, which means that the risk of bias is difficult to judge. In particular, it is difficult to assess the quality of the data used (and therefore the validity of study results), due to the lack of reporting around this, including how parameters were identified. These factors indicate uncertainty about the robustness of the overall result that vaccination is cost-effective.

\section{Intervention and comparator}

Three studies directly compared two vaccine types and found that the cost-effectiveness of vaccination varied by type of vaccine. ${ }^{23-25}$ Six studies included a comparison of a strategy to vaccinate older people to no vaccination strategy. ${ }^{23} 2426$ 28-31 Of these studies, the comparator arm included a proportion of participants who would be vaccinated which ranged from $0 \%$ to $27 \%$ in low- or mixed-risk populations and $68 \%$ in a high-risk population. The reported coverage rates for the countries included in the studies ranged from $12 \%$ to $76 \%$. The data indicate that practice varies between countries and that the decision to implement or change a vaccination strategy for older people needs to take the underlying coverage rate into account. Two studies considered a vaccination level very similar to reality, improving the validity of these studies. ${ }^{24}{ }^{29}$ One study included antiviral drugs as a comparator but did not directly compare them to a vaccination intervention arm and so results are not presented. $^{31}$

Four studies, which synthesised evidence from multiple sources within an economic model, did not report vaccination type (eg, adjuvant, quadrivalent, etc)..$^{24} 283031$ As there are different vaccination types available, it would be more robust if evaluations focused on a specific type; in these cases, it is not known whether data may have been synthesised from studies for different vaccination types, reducing validity. 


\section{Effectiveness data}

One study was conducted alongside an RCT. ${ }^{26}$ This was single blinded only as double blinding was not feasible due to the intervention type (injection), which may introduce bias. One study identified the efficacy inputs from prospective cohort studies which were not randomised or blinded to intervention, increasing the risk of bias. ${ }^{23}$ The authors did not report detailed information about the cohort study design or participants, so it is not possible to assess the validity and robustness of the data and analyses used in the economic model. The remaining studies synthesised data about vaccine efficacy/effectiveness from several sources. None of these economic evaluations reported the methods used to identify and select studies to estimate effectiveness data inputs. Most of the authors reported that they needed to supplement data on vaccine efficacy with evidence from other countries or apply the same efficacy values across different countries in the same study. ${ }^{242528-31}$ This means that it is not possible to assess the validity and robustness of the vaccine efficacy/effectiveness data used in the economic models. For example, Scuffham and West $^{31}$ used the same efficacy source for three countries, and many of the papers use the same network meta-analysis for effectiveness of the influenza vaccination. This reduces the validity of study results and suggests that the studies may be over-representing a limited evidence base.

\section{Measure of health benefit}

Four studies undertook a cost-effectiveness analysis alone, with the measure of health benefit provided in terms of life-years gained or deaths averted, 23252831 two studies performed a cost-utility analysis with quality-adjusted life-years (QALYs) as the measure of health benefit ${ }^{24} 30$ and two studies included both cost-effectiveness and costutility analyses. ${ }^{26} 29$ All studies assessed life-years gained as a result of avoiding death related to influenza complications. In the four studies reporting cost-utility results, a single study accounted for morbidity by applying lost utility related to mortality to estimate QALYs. ${ }^{26}$ The other studies also accounted for short-term changes in utility as a result of having influenza. ${ }^{24} 2930$

The robustness of QALY estimates is questionable due to the limited relevant evidence and small study samples of utility studies. A review concluded that no utility studies using robust methods were available for influenza, which was reflected in the cost-utility evaluations identified. $^{35}$ Lugner et $a l^{30}$ took utility decrements relating to influenza from an English postal survey of 288 patients with confirmed influenza, using the European Quality of Life 5-Dimension Health Questionnaire. ${ }^{30}$ The generalisability of this study to the elderly Dutch population is difficult to assess as patient characteristics were not reported. Meier et $a l^{24}$ and Brydak et $a l^{29}$ applied utility values taken from a survey of 15 working age healthcare workers in the USA with utilities scored using the health utilities index. ${ }^{24} 2937$ This is arguably not relevant to the elderly population. The WHO guidelines recommend that economic evaluations are cost-utility analyses; however, without robust utility data, cost-utility evaluations will inevitably have limited validity. ${ }^{32}$

ILI was used in all studies as a proxy for influenza. The severity and subsequent cost of influenza can vary substantially; therefore, there are concerns that this outcome is too broad. ${ }^{38}$ However, this is the primary end point of clinical trials, and subsequently, studies are restricted by this. ${ }^{38}$ Longer term outcomes associated with influenza were not considered, likely due to a lack of robust clinical data and the impact of confounding factors in the long term. ${ }^{11}$ The likely direction of bias caused by excluding long-term outcomes is unclear; it may understate the value of vaccination if it increases a person's health over time or may overstate the value of vaccination if side effects occur in the long term.

\section{Costs}

Seven out of eight studies used a 1-year time horizon for costs, consistent with an influenza season and the shortterm/immediate associated costs; therefore, discounting costs were irrelevant. $^{23} 252628-31$ The remaining study considered repeat vaccinations and costs each influenza season over a lifetime. ${ }^{24}$ Where relevant, studies applied country-specific guidelines for discounting outcomes, ranging from $1.5 \%$ to $5 \%$ annually.

The majority of studies considered direct costs only. This approach is justified as indirect costs are less important in a retired population. Societal costs (eg, over-the-counter drugs) are likely to be minimal due to the nature of the illness. Appropriately, the only study that included productivity losses did so because they considered herd immunity effects in the working age population; the study also included a scenario in which only direct costs were considered. ${ }^{30}$ All studies included costs associated with purchasing vaccines, influenza-related treatments, primary care visits and hospitalisations. Six studies included administration costs. ${ }^{25} 2628-31$ The total cost of vaccination varied from $€ 8.50$ to $€ 35.95$ per person, and differences between studies mainly occurred due to variation in administration costs rather than variation in vaccine cost; lower costs were associated with opportunistic vaccination. Transportation costs, over-the-counter medication costs and private nursing home costs were considered in the societal perspective of one study. ${ }^{24}$ A single study included vaccination side effect costs; in the short term, these are rare and usually require only minimal medical attention. ${ }^{31}$ The inclusion of side effect costs did not have a significant effect on total costs or overall ICER results in this study (supported by a sensitivity analysis). ${ }^{31}$ Longer term evidence is lacking, with the majority of studies in the elderly population having short time horizons; hence, data on longer term side effects would not be identified and could not have been included by studies; in the future if studies expand their time horizons, this may have implications on results. ${ }^{11}$ Only one study applied costs to promote vaccination. ${ }^{26}$ No studies considered 
the cost of service redesign to increase vaccination (eg, opening additional clinics) which may be relevant for healthcare systems with restraints on resources available (eg, in the case of an ageing population). Thus, the true cost of vaccination is likely to be underestimated across studies depending on the healthcare setting.

\section{Model design}

On the whole, modelling approaches were relatively simple (eg, static decision tree models), reflecting gaps in the evidence base and the short time horizon captured in effectiveness studies. As the main outcomes of the vaccination occur over a single influenza season and occur once only during this time (ie, influenza infection or no infection), decision trees are a clear and logical structure for the model and are likely to be sufficient to capture the majority of health outcomes and costs. One study used a dynamic transmission model and was therefore able to capture the impact of transmission and herd immunity. ${ }^{30}$ This characterises interactions across the population and is therefore more reflective of the spread of disease and the indirect benefit for those not vaccinated. Overall, this model was reported well and seemed to be robust, but it did require more data than the simpler model approaches. The authors in this case had access to data on population interactions, but this may not be available in every country. Models that exclude herd immunity are likely to underestimate the cost-effectiveness of influenza vaccination; however, WHO guidelines recommend that a static model is acceptable as a conservative approach. ${ }^{32}$

More complex methodologies may capture the nature of the disease more accurately; however, given the noted lack of clinical evidence, it is unlikely that the current evidence base is sufficient to inform these ${ }^{11}$ For example, patient-level simulations can also be more precise when modelling population interactions. ${ }^{39}$ Additionally, stochastic models, with events occurring randomly, can more realistically model epidemics, as these occur by chance of transmission among population interactions. ${ }^{20}$

\section{Methods to address uncertainty}

Most scenarios tested in sensitivity analysis still resulted in favourable cost-effectiveness results for influenza vaccination. However, there were limitations to the range of sensitivity analyses reported, given that many of the studies recognised that there are issues with the evidence base. Methods used to analyse uncertainty were varied, such that results are not comparable. The WHO recommends that sensitivity analyses for economic evaluations of vaccines should vary the following five parameters as a minimum: discount rates, vaccine efficacy, influenza incidence, influenza complication rates and vaccine price. ${ }^{32}$ None of the studies identified varied all of the recommended parameters, and because of this, we cannot fully assess the robustness of the results. One of the studies performed a comprehensive one-way sensitivity analysis, presenting a resulting tornado diagram. ${ }^{29}$ One study did not perform any one-way/scenario analysis. ${ }^{23}$ The remaining studies chose to vary a limited selection of key variables but did not explain the rationale for these. ${ }^{24-26} 283031$ Three studies provided and justified ranges used in sensitivity analyses. ${ }^{24} 2529$ The remainder detailed the ranges but did not provide justification, which means that validity cannot be assessed. ${ }^{26} 283031$ Three studies included probabilistic sensitivity analysis, but one did not report the results restricted to the elderly population alone. ${ }^{2324} 29$ This lack of thorough investigation and transparency restricts our ability to assess the robustness of the results or to explore the transferability of study results to alternative countries, settings and influenza seasons.

\section{DISCUSSION}

The majority (seven of eight) of the identified studies found influenza vaccination to be cost-effective in an elderly European population. The studies indicated some differences what vaccination strategy may be cost-effective. Adjuvant or quadrivalent vaccinations had more favourable results compared with standard vaccinations. Vaccination targeted to high-risk groups of older people were generally more cost-effective, and passive vaccination strategies appeared more cost-effective than opportunistic strategies. Decision makers using this evidence would need to check which papers are most relevant to their research question.

Most scenarios tested in sensitivity analysis still resulted in favourable cost-effectiveness results for influenza vaccination. In addition, the studies that conducted probabilistic sensitivity analysis concluded that the likelihood of cost-effectiveness given parameter uncertainty was high. Results were sensitive to variations in the strain of influenza incidence, vaccination type, efficacy and strategy, population risk and modelling characteristics. Review findings are in alignment with the previous review identified. $^{40}$

\section{Limitations of the evidence base}

The robustness of conclusions about the cost-effectiveness of vaccination in older people is limited due to the evidence gaps and subsequent uncertainty demonstrated in the literature base, as well as the limited investigation into this uncertainty. Studies did not report all of the information needed to assess the internal and external validity of results. Robust economic evaluations require high-quality evidence of effectiveness, service use and health benefit. In the studies reviewed here, the data used are uncertain and in the case of the effectiveness data reliant on underpowered trials. Most of the studies reported limited information about the methods used to identify and select effectiveness data, the relevance of the data to the elderly population or the quality of the data used to estimate effectiveness parameters. In addition, the evidence base is small, especially considering the number of questions decision makers could have. Many of the conclusions about cost-effectiveness rely on the results of single studies. ${ }^{24} 31$ 
The studies reported limited sensitivity analyses to test the robustness of the conclusions to the assumptions made or uncertainty in the data used. There was also a lack of reporting on key items such as the rationale for the data ranges used in sensitivity analysis. Detailed and clearly justified sensitivity analysis with robust ranges is needed to identify the level of uncertainty and draw evidence-based conclusions. ${ }^{11}$ Further investigation into uncertainty, in particular, probabilistic sensitivity analysis that includes all parameters, is needed to estimate the level of certainty of the cost-effectiveness results.

\section{Limitations of this review}

The review focused on the elderly population, which is one of many high-risk groups. To prioritise the right groups to target for vaccination, due to cost and resource constraints, decision makers are likely to need to conduct further reviews of the economic evidence for other highrisk groups. For example, these analyses might focus on groups with chronic conditions, pregnant women or children. This would produce a more balanced judgement of where to prioritise intervention.

The original search for studies used a single database (NHS EED), increasing the chance of missing papers that could change the conclusions of the review. However, at the time, NHS EED was comprehensive, searching a wide range of databases, and was regularly updated. The updated search included more databases. The review included English-language articles only, which risks language bias. Searches were limited to published journal articles. Widening the searches to the grey literature or unpublished reports may have been more likely to identify studies with inconclusive or negative cost-effectiveness results. ${ }^{41}$ The generalisability of some of the results of this review to other settings (outside the EU) may be limited, due to inevitable differences resource use, treatment pathways and population characteristics.

\section{Future research}

This review highlights several areas that would benefit from additional research. Parameter uncertainty affected the strength of economic evaluations, largely because many of the trials for vaccine efficacy are underpowered, non-randomised and susceptible to bias, ${ }^{11}$ and deterministic scenarios were not explored in thorough scenario analyses. A number of the evaluations used the same effectiveness data to estimate model parameters; this will over-represent a limited evidence base. Without more and stronger quality data to inform parameters, economic evaluations will always be subject to a high degree of uncertainty. Trials for the vaccination cover the influenza season, typically a 6 -month period over winter. ${ }^{11} \mathrm{~A}$ predominance of short-term evidence in the literature prevented the identified studies from including longer term outcomes. There was also a lack of evidence for each country, with studies relying on external data sources with questionable relevance to their population. Moreover, this evidence gap meant that many studies used the same sources for certain inputs, potentially over-representing the results of a small and low-quality evidence base. Work to ensure that robust country-relevant data inputs are available would increase the validity of future evaluations. Increasing this evidence base could lead policy makers to revaluate current recommendations.

The body of economic evidence suggests that influenza vaccination of elderly populations may be cost-effective, but data, methodological transparency and exploration of uncertainty are lacking. Baguelin $e t a l^{36}{ }^{42}$ developed a complex epidemiological model to explore the relative cost-effectiveness of vaccination in different low- and highrisk groups. While these evaluations give an indication of the value of complex models, both studies also faced limitations in data about the effectiveness of vaccines and estimates of service use and health benefits. ${ }^{364}$

Future economic evaluations should follow good practice guidelines and ensure that they are transparently reported to assist reviewers in the future. Economic evaluations integrated into long-term, prospective, controlled studies (eg, observational cohort studies or RCTs) or prospective study in elderly populations would help to address the evidence gaps around effectiveness, service use and health benefit. Since the influenza virus mutates each season and differences in vaccine matching each season, a combination of multiple studies and those conducted over several influenza seasons are needed. Improvements in the evidence base would support the development and analysis of more sophisticated modelling techniques. Such models could then characterise the epidemiology of influenza and complications in elderly people and incorporate the impact of herd immunity within the elderly population and between age groups. Robust data about service use and health benefits would allow more detailed estimates of the potential for different vaccination strategies for older people to be cost-effective in different settings.

Correction notice This paper has been amended since it was published Online First. Owing to a scripting error, some of the publisher names in the references were replaced with 'BMJ Publishing Group'. This only affected the full text version, not the PDF. We have since corrected theseerrors and the correct publishers have been inserted into the references.

Acknowledgements The research was completed as part of a master's degree dissertation (Master of Public Health) at the University of Manchester. The authors would like to thank the reviewers of the original dissertation for their helpful comments and suggestions.

Contributors GES and JE conducted the literature search with oversight from LMD. GES wrote the first draft of the manuscript. JE and LMD contributed to the final writing of the paper.

Competing interests None declared.

Provenance and peer review Not commissioned; externally peer reviewed.

Data sharing statement Search strategies and data extraction templates are available in the supplementary material. No other unpublished data from the study are available.

Open Access This is an Open Access article distributed in accordance with the Creative Commons Attribution Non Commercial (CC BY-NC 4.0) license, which permits others to distribute, remix, adapt, build upon this work non-commercially, and license their derivative works on different terms, provided the original work is properly cited and the use is non-commercial. See: http://creativecommons.org/ licenses/by-nc/4.0/ 
(C) Article author(s) (or their employer(s) unless otherwise stated in the text of the article) 2017. All rights reserved. No commercial use is permitted unless otherwise expressly granted.

\section{REFERENCES}

1. Szucs TD. Health economic research on vaccinations and immunisation practices - an introductory primer. Vaccine 2005;23:2095-103.

2. World Health Organisation. Influenza (Seasonal). 2015. http://www. who.int/mediacentre/factsheets/fs211/en/ (accessed 17 Nov 2015)

3. Monto AS. Seasonal influenza and vaccination coverage. Vaccine 2010;28 Suppl 4(Suppl 4):D33-D44.

4. Preaud E, Durand L, Macabeo B, et al. Annual public health and economic benefits of seasonal influenza vaccination: a European estimate. BMC Public Health 2014;14:813.

5. Mauskopf J, Klesse M, Lee S, et al. The burden of influenza complications in different high-risk groups: a targeted literature review. J Med Econ 2013;16:264-77.

6. Weiskopf D, Weinberger B, Grubeck-Loebenstein B. The aging of the immune system. Transp/ Int 2009;22:1041-50.

7. Meier CR, Napalkov PN, Wegmüller Y, et al. Population-based study on incidence, risk factors, clinical complications and drug utilisation associated with influenza in the United Kingdom. Eur J Clin Microbiol Infect Dis 2000;19:834-42.

8. Eurostat. Population age structure by major age groups, 2005 and 2015 (\% of the total population). http://ec.europa.eu/eurostat/ statistics-explained/index.php/File:Population age structure by major_age_groups,_2005_and_2015_(\%25_of_the_total_population) YB16.png (accessed 7 Dec 2016).

9. Hardelid P, Pebody R, Andrews N. Mortality caused by influenza and respiratory syncytial virus by age group in England and Wales 19992010. Influenza Other Respir Viruses 2013;7:35-45.

10. Simonsen L, Viboud C, Taylor R, et al. The epidemiology of influenza and its control. In: Rappuoli R, Del Giudice G, eds. Influenza Vaccines for the Future SE - 2. Springer Basel, 2011:27-54. doi.

11. Jefferson T, Di Pietrantonj C, Al-Ansary LA, et al. Vaccines for preventing influenza in the elderly. Cochrane Database Syst Rev 2010:CD004876.

12. The Council of the European Union.. Council Recommendation of 22 December 2009 on seasonal influenza vaccination.. 2009. http:// www.epha.org/IMG/pdf/Council_Reccomendation_on_seasonal_flu vaccine.pdf (accessed 20 Nov 2015).

13. European Centre for Disease Prevention and Control. Seasonal influenza vaccination in Europe. Overview of vaccination recommendations and coverage rates in the EU Member States for the 2012-13 influenza season. 2015.

14. Osterholm MT, Kelley NS, Sommer A, et al. Efficacy and effectiveness of influenza vaccines: a systematic review and metaanalysis. Lancet Infect Dis 2012;12:36-44.

15. Haq K, McElhaney JE. Immunosenescence: Influenza vaccination and the elderly. Curr Opin Immunol 2014;29:38-42.

16. Jefferson T, Rivetti D, Rivetti A, et al. Efficacy and effectiveness of influenza vaccines in elderly people: a systematic review. Lancet 2005;366:1165-74.

17. Nixon J, Stoykova B, Glanville J, et al. The U.K. NHS economic evaluation database. Economic issues in evaluations of health technology. Int J Technol Assess Health Care 2000;16:731-42 http:// www.ncbi.nlm.nih.gov/pubmed/11028129.

18. Centre for Reviews and Dissemination. Search strategies. Univ. York 2014 http://www.crd.york.ac.uk/crdweb/searchstrategies.asp (accessed 21 Jul 2016).

19. Centre for Reviews and Dissemination. NHS Economic Evaluation Database (NHS EED) Handbook 2007 http://www.york.ac.uk/inst// crd/pdf/nhseed-handbook2007.pdf (accessed 18 Dec 2015).

20. Kim SY, Goldie SJ. Cost-effectiveness analyses of vaccination programmes : a focused review of modelling approaches. Pharmacoeconomics 2008;26:191-215 http://www.ncbi.nlm.nih.gov/ pubmed/18282015.

21. OECD. Prices - Inflation (CPI) - OECD Data. 2015. https://data.oecd. org/price/inflation-cpi.htm (accessed 18 Dec 2015).
22. OECD. Purchasing Power Parities (PPPs) Data. 2015. http://www.o ecd.org/std/prices-ppp/purchasingpowerparitiespppsdata.htm (accessed 18 Dec 2015)

23. Baio G, Pammolli F, Baldo V, et al. Object-oriented influence diagram for cost-effectiveness analysis of influenza vaccination in the Italian elderly population. Expert Rev Pharmacoecon Outcomes Res 2006;6:293-301.

24. Meier G, Gregg M, Poulsen Nautrup B. Cost-effectiveness analysis of quadrivalent influenza vaccination in at-risk adults and the elderly: an updated analysis in the U.K. J Med Econ 2015;18:746-61.

25. Piercy J, Ryan J, Megas F. Economic evaluation of MF59 adjuvanted vaccine against influenza in the high-risk elderly population in France. J Med Econ http://www.tandfonline.com/doi/abs/10.3111/ 200407001018\#.VktWiXbhDIU (accessed 17 Nov 2015).

26. Allsup S, Haycox A, Regan M, et al. Is influenza vaccination cost effective for healthy people between ages 65 and 74 years? A randomised controlled trial. Vaccine 2004;23:639-45.

27. Jit M, Newall AT, Beutels P. Key issues for estimating the impact and cost-effectiveness of seasonal influenza vaccination strategies. Hum Vaccin Immunother 2013;9:834-40.

28. Postma MJ, Bos JM, van Gennep M, et al. Economic evaluation of influenza vaccination. Assessment for The Netherlands. Pharmacoeconomics 1999;16(Suppl 1):33-40 http://www.ncbi.nlm. nih.gov/pubmed/10623374

29. Brydak L, Roiz J, Faivre P, et al. Implementing an influenza vaccination programme for adults aged $\geq 65$ years in Poland: a costeffectiveness analysis. Clin Drug Investig 2012;32:73-85.

30. Lugnér AK, van Boven $M$, de Vries $R$, et al. Cost effectiveness of vaccination against pandemic influenza in European countries: mathematical modelling analysis. BMJ 2012;345:e4445.

31. Scuffham PA, West PA. Economic evaluation of strategies for the control and management of influenza in Europe. Vaccine 2002;20:2562-78 http://www.ncbi.nlm.nih.gov/pubmed/12057614.

32. Walker DG, Hutubessy R, Beutels P. WHO Guide for standardisation of economic evaluations of immunization programmes. Vaccine 2010;28:2356-9.

33. Drummond MF, Jefferson TO. Guidelines for authors and peer reviewers of economic submissions to the BMJ. The BMJ Economic Evaluation Working Party. BMJ 1996;313:275-83 http://www. pubmedcentral.nih.gov/articlerender.fcgi?artid=2351717\&tool= pmcentrez\&rendertype=abstract.

34. Philips Z, Ginnelly L, Sculpher M, et al. Review of guidelines for good practice in decision-analytic modelling in health technology assessment. Health Technol Assess 2004;8:1-158 http://www.ncbi. nlm.nih.gov/pubmed/15361314.

35. van Hoek AJ, Underwood A, Jit M, et al. The impact of pandemic influenza $\mathrm{H} 1 \mathrm{~N} 1$ on health-related quality of life: a prospective population-based study. PLoS One 2011;6:e17030.

36. Baguelin $M$, Hoek $A J$, Jit $M$, et al. Vaccination against pandemic influenza $\mathrm{A} / \mathrm{H} 1 \mathrm{~N} 1 \mathrm{v}$ in England: a real-time economic evaluation. Vaccine 2010;28:2370-84.

37. Rothberg MB, He S, Rose DN. Management of influenza symptoms in healthy adults. J Gen Intern Med 2003;18:808-15 http://www. pubmedcentral.nih.gov/articlerender.fcgi?artid=1494927\&tool= pmcentrez\&rendertype=abstract.

38. World Health Organisation. World Health Organization. Global epidemiological surveillance standards for influenza 2013 http://www. who.int/influenza/resources/documents/WHO_Epidemiological Influenza_Surveillance_Standards_2014.pdf?ua=1 (accessed 7 Jan 2016)

39. Davis S, Stevenson M, Tappenden P, et al. NICE DSU Technical Support Document 15: cost-effectiveness modelling using patientlevel simulation. Rep BY Decis Support UNIT 2014 http://www. nicedsu.org.uk/TSD15_Patient-level_simulation.pdf (accessed 7 Jan 2016).

40. Postma MJ, Baltussen RP, Palache AM, et al. Further evidence for favorable cost-effectiveness of elderly influenza vaccination. Expert Rev Pharmacoecon Outcomes Res 2006;6:215-27.

41. Bell CM, Urbach DR, Ray JG, et al. Bias in published cost effectiveness studies: systematic review. BMJ 2006;332:699-703.

42. Baguelin M, Camacho A, Flasche S, et al. Extending the elderly- and risk-group programme of vaccination against seasonal influenza in England and Wales: a cost-effectiveness study. BMC Med 2015;13:236 